\title{
Reduced intrahepatic fat content is associated with increased whole-body lipid oxidation in patients with type 1 diabetes
}

Received: 9 June 2005 / Accepted: 21 July 2005 / Published online: 1 November 2005

(C) Springer-Verlag 2005

\begin{abstract}
Aims/hypothesis: Insulin resistance may be associated with ectopic fat accumulation potentially determined by reduced lipid oxidation. In patients with type 1 diabetes peripheral insulin resistance is associated with higher intramyocellular lipid content. We assessed whether these patients are also characterised by intrahepatic fat accumulation and abnormal fat oxidation. Methods: Nineteen
\end{abstract}

G. Perseghin $(\bowtie) \cdot$ G. Lattuada $\cdot$ F. Costantino $\cdot$ L. Luzi Internal Medicine, Section of Nutrition/Metabolism, via Olgettina 60, 20132 Milan, Italy

e-mail: perseghin.gianluca@hsr.it

Tel.: +39-02-26432614

Fax: +39-02-26432771

G. Perseghin · F. De Cobelli · P. Scifo ·

A. Del Maschio - L. Luzi

Unit of Clinical Spectroscopy, IRCCS H, San Raffaele, Milan, Italy

G. Perseghin · L. Luzi

Centre 'Physical Exercise for Health and Wellness', Milan, Italy

F. De Cobelli - A. Esposito - T. Canu - A. Del Maschio Division of Diagnostic Radiology, IRCCS H, San Raffaele, Milan, Italy

P. Scifo

Division of Nuclear Medicine, IRCCS H, San Raffaele, Milan, Italy

F. De Taddeo · P. Maffi · A. Secchi

Internal Medicine - Section of Organ Transplantation, IRCCS H, San Raffaele,

Milan, Italy

A. Secchi · A. Del Maschio

Vita-Salute San Raffaele University,

Milan, Italy

L. Luzi

Faculty of Exercise Sciences, University of Milan,

Milan, Italy patients with type 1 diabetes ( 6 women, 13 men, age $35 \pm 7$ years, BMI $23 \pm 3 \mathrm{~kg} / \mathrm{m}^{2}, \mathrm{HbA}_{1} \mathrm{c} 8.7 \pm 1.4 \%$ ) and 19 healthy matched individuals were studied by (1) euglycaemic-hyperinsulinaemic clamp combined with $\left[6,6-{ }^{2} \mathrm{H}_{2}\right]$ glucose infusion to assess whole-body glucose metabolism; (2) indirect calorimetry to assess glucose and lipid oxidation; and (3) localised ${ }^{1} \mathrm{H}$-magnetic resonance spectroscopy of the liver to assess intrahepatic fat content. Results: Patients with type 1 diabetes showed a reduced insulin-stimulated metabolic clearance rate of glucose $\left(4.3 \pm 1.3 \mathrm{ml} \mathrm{kg}_{-1}^{-1} \min _{-1}^{-1}\right)$ in comparison with normal subjects $\left(6.0 \pm 1.6 \mathrm{ml} \mathrm{kg}^{-1} \mathrm{~min}^{-1}\right.$; $p<0.001)$. Endogenous glucose production was higher in diabetic patients $(p=0.001)$ and its suppression was impaired during insulin administration (66 \pm 30 vs $92 \pm 8 \%$; $p=0.047$ ) in comparison with normal subjects. Plasma glucagon concentrations were not different between groups. The estimated hepatic insulin concentration was lower in diabetic patients than in normal subjects $(p<0.05)$, as was the intrahepatic fat content $(1.5 \pm 0.7 \%$ and $2.2 \pm 1.0 \%$ respectively; $p<0.03$ ), the latter in association with a reduced respiratory quotient $(0.74 \pm 0.05$ vs $0.84 \pm 0.06 ; p=0.01)$ and increased fasting lipid oxidation ( $1.5 \pm 0.5$ vs $0.8 \pm 0.4 \mathrm{mg} \mathrm{kg}^{-1}$ $\left.\min ^{-1} ; p<0.01\right)$. Conclusions/interpretation: In patients with type 1 diabetes, insulin resistance was not associated with increased intrahepatic fat accumulation. In fact, diabetic patients had reduced intrahepatic fat content, which was associated with increased fasting lipid oxidation. The unbalanced hepatic glucagon and insulin concentrations affecting patients with type 1 diabetes may be involved in this abnormality of intrahepatic lipid metabolism.

Keywords Ectopic fat - Insulin resistance - Intrahepatic fat content $\cdot$ Lipid oxidation $\cdot$ Magnetic resonance spectroscopy

Abbreviations IHF: intrahepatic fat - IMCL: intramyocellular lipid · MRS: magnetic resonance spectroscopy $\cdot$ PPM: parts per million 


\section{Introduction}

Ectopic fat accumulation within the skeletal muscle has been hypothesised to be the pathogenic event responsible for the development of peripheral insulin resistance [1]. Increased intramyocellular lipid content (IMCL) was reported in association with insulin resistance in normal humans [2], individuals with an increased risk of developing type 2 diabetes [3] and in patients with overt type 2 diabetes [4]. Similarly, ectopic fat accumulation within the liver has been reported in association with impairment of insulinstimulated glucose metabolism, of the suppression of endogenous glucose production, and of whole-body lipolysis in individuals with non-alcoholic fatty liver disease [5]. Recently, intrahepatic fat (IHF) content has been assessed as a continuous variable by means of ${ }^{1} \mathrm{H}$-magnetic resonance spectroscopy (MRS), and was reported to be associated with hepatic insulin resistance in non-diabetic individuals [6].

It has also been shown that, in patients with type 1 diabetes, as in patients with type 2 diabetes, peripheral and hepatic insulin resistance may be associated with higher IMCL content [4] and that these patients have a greater reliance on lipid than on carbohydrate oxidation to maintain energy homeostasis in the resting condition [7], during moderate exercise [8] and after strenuous exercise [9].

Hypothesising that ectopic fat accumulation may be associated with impairment of insulin action at both the skeletal muscle and the liver site, and based on the hypothesis that hepatic fat content may be influenced by lipid oxidation, the present study was undertaken to assess whether moderately insulin-resistant patients with type 1 diabetes are characterised by abnormal IHF accumulation.

\section{Subjects, materials and methods}

\section{Subjects}

Nineteen patients with type 1 diabetes and 19 healthy individuals were recruited in the outpatient services of the Diabetes Unit and of the Centre of Nutrition/Metabolism of the Scientific Institute H. San Raffaele, Milan. Among the diabetic patients, two had proliferative retinopathy, nine had non-proliferative retinopathy and five had sensorymotor neuropathy. Normal subjects were comparable to patients with type 1 diabetes for anthropometric features and lifestyle habits. Habitual physical activity was assessed using a questionnaire [10]. Body weight was stable for at least 6 months and the women were not taking oral contraceptive steroids. A history of hepatic disease, substance abuse or daily consumption of more than one alcoholic drink daily $(<20 \mathrm{~g} /$ day $)$ or the equivalent in beer and wine were exclusion criteria. The anthropometric characteristics of the subjects are summarised in Table 1. All subjects were in good health, as assessed by medical history, physical examination, haematological analysis and urinalysis. Informed consent was obtained from all subjects after ex-
Table 1 Anthropometric and laboratory characteristics of the study groups

\begin{tabular}{lll}
\hline & $\begin{array}{l}\text { Type 1 diabetes } \\
\text { mellitus }\end{array}$ & $\begin{array}{l}\text { Normal } \\
\text { subjects }\end{array}$ \\
\hline Number of men, women & 13,6 & 13,6 \\
Age (years) & $35 \pm 7$ & $34 \pm 6$ \\
BMI $\left(\mathrm{kg} / \mathrm{m}^{2}\right)$ & $22.7 \pm 2.8$ & $23.0 \pm 3.1$ \\
Diabetes duration (years) & $21 \pm 6$ & - \\
Daily insulin dose (s.c. U/day) & $52 \pm 13$ & - \\
Physical activity index & $8.9 \pm 1.3$ & $8.2 \pm 1.9$ \\
Plasma glucose (mmol/l) & $11.1 \pm 1.2^{\mathrm{a}}$ & $4.8 \pm 0.5$ \\
HbA ${ }_{1} \mathrm{c}(\%)$ & $8.7 \pm 1.4^{\mathrm{a}}$ & $5.6 \pm 0.6$ \\
Creatinine ( $\mu$ mol/1) & $70 \pm 15$ & $72 \pm 18$ \\
Creatinine clearance (ml/s) & $89 \pm 19$ & $99 \pm 24$ \\
Plasma NEFA (mmol/1) & $0.85 \pm 0.22^{\mathrm{a}}$ & $0.49 \pm 0.22$ \\
Total cholesterol (mmol/l) & $4.78 \pm 0.88$ & $4.53 \pm 0.55$ \\
HDL cholesterol (mmol/l) & $1.32 \pm 0.36$ & $1.22 \pm 0.31$ \\
LDL cholesterol (mmol/l) & $3.03 \pm 0.78$ & $2.89 \pm 0.57$ \\
Triglycerides (mmol/l) & $1.04 \pm 0.56$ & $0.88 \pm 0.39$ \\
Aspartate aminotransferase (U/1) & $22 \pm 7$ & $24 \pm 5$ \\
Alanine aminotransferase (U/l) & $24 \pm 14$ & $24 \pm 8$ \\
$\gamma$-Glutamyltransferase (U/l) & $22 \pm 13$ & $17 \pm 5$ \\
\hline
\end{tabular}

${ }^{\mathrm{a}} p<0.01$ vs normal subjects (independent two-tailed $t$-test)

planation of the purposes, nature and potential risks of the study. The protocol was approved by the Ethical Committee of the Scientific Institute H. San Raffaele.

\section{Experimental protocol}

Subjects were instructed to consume an isocaloric diet and to abstain from exercise activity for 3 weeks before the studies. Women were studied between days 3 and 8 of the menstrual cycle. Subjects were studied by means of the euglycaemic-hyperinsulinaemic clamp to assess wholebody insulin sensitivity after a 10 -h overnight fast. Within 2-3 days they were studied by means of ${ }^{1} \mathrm{H}-\mathrm{MRS}$ in order to assess IHF content. The MRS session was performed in the Division of Diagnostic Radiology of our Institute between 07.00 and 09.00 hours after a 10-h overnight fast. Because lipid oxidation and the IHF content may be affected differently in the fasting condition by an overnight insulin infusion and in order to study the patients with diabetes as they may be in everyday life, they were instructed to undergo the usual therapeutic regimen the day before the studies and to abstain from any s.c. insulin only in the morning of the clamp and MRS studies.

\section{Euglycaemic-hyperinsulinaemic clamp}

Patients were admitted to the Centre of Nutrition/Metabolism of our Institute the night before the clamp study. During the night, when capillary glucose concentration exceeded $13 \mathrm{mmol} / \mathrm{l}$, an exogenous insulin infusion $(0.1 \mathrm{mU}$ $\left.\mathrm{kg}^{-1} \mathrm{~min}^{-1}\right)$ was started. Normal subjects were admitted at 
07.00 hours after a 10-h overnight fast. A Teflon catheter was inserted into an antecubital vein for infusions and an additional one was inserted retrogradely into a wrist vein for blood sampling. The hand was kept in a heated box throughout the experiment to allow sampling of arterialised venous blood. A bolus of $\left[6,6-^{2} \mathrm{H}_{2}\right]$ glucose $(5 \mathrm{mg} / \mathrm{kg}$ body weight) was administered to normal subjects; the bolus administered to diabetic patients was adjusted as described previously [11] according to the fasting plasma glucose concentration. Immediately after the administration of the bolus a continuous infusion $(0.05 \mathrm{mg} / \mathrm{kg}$ body weight per minute) of $\left[6,6-{ }^{2} \mathrm{H}_{2}\right]$ glucose (Masstrace, Woburn, MA, USA) was started. Blood samples for postabsorptive plasma glucose, total cholesterol, HDL cholesterol, triglycerides, NEFA and free insulin were performed in duplicate in the postabsorptive condition. Following a 150-min tracer equilibration period, a euglycaemic-hyperinsulinaemic clamp was performed as described previously [4]. Insulin was infused at $40 \mathrm{mU} \mathrm{m} \mathrm{min}^{-1}$ and plasma glucose concentration was kept at $\sim 5 \mathrm{mmol} / 1$ for additional $150 \mathrm{~min}$ by means of a variable infusion of $20 \%$ dextrose infusion. When fasting hyperglycaemia was present in patients with type 1 diabetes, we attempted to reduce it and maintain it close to the euglycaemic level (Fig. 1). Blood samples for plasma hormones, substrates and tracer enrichment were drawn every 15 min throughout the study.

\section{Indirect calorimetry}

Indirect calorimetry was performed continuously, while the subject was lying quietly, for $45 \mathrm{~min}$ during the basal equilibration period and at the end of the clamp, using a ventilated hood system (2900 Metabolic Measurement Cart; Sensormedics, Milan, Italy) to measure volume of oxygen utilisation and carbon dioxide output in order to calculate glucose and lipid oxidation. The mean $\mathrm{CV}$ within the session was below $2 \%$ for measurements of both $\mathrm{O}_{2}$ and $\mathrm{CO}_{2}$.

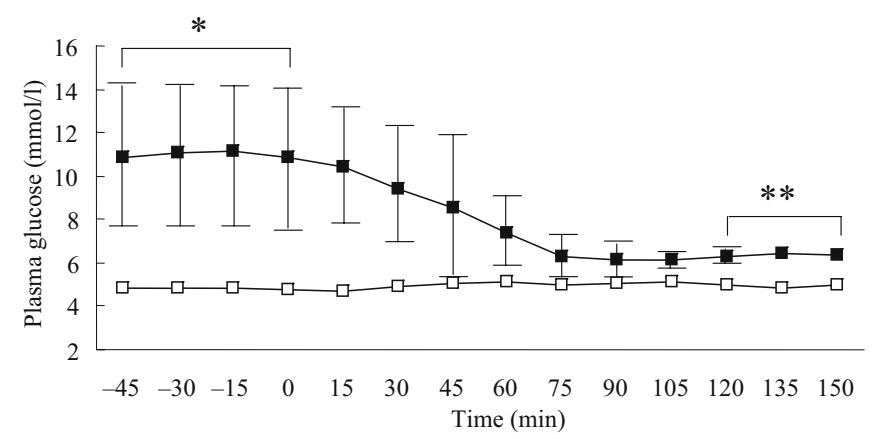

Fig. 1 Plasma glucose profile during the fasting and clamp conditions in patients with type 1 diabetes (filled symbols) and normal subjects (open symbols). ${ }^{*} p<0.01,{ }^{*} p<0.05$ vs normal subjects (independent $t$-test)
Hepatic ${ }^{1} \mathrm{H}$-magnetic resonance spectroscopy

${ }^{1} \mathrm{H}-\mathrm{MRS}$ was performed at rest and with the patients in the supine position with the use of a $1.5 \mathrm{~T}$ whole-body scanner (Gyroscan Intera Master 1.5 MR System; Philips Medical Systems, Best, The Netherlands) using a conventional circular superficial coil ( $\mathrm{C} 1$ coil). First, coronal and transverse images of the liver were obtained in all patients. Then, T1 in-phase and out-of-phase sequences were obtained to look for a potential loss of signal on out-ofphase images, indicating the presence of IHF accumulation. Next an $8-\mathrm{cm}^{3}$ spectroscopic volume of interest was positioned within the right lobe, avoiding major blood vessels, intrahepatic bile ducts and the lateral margin of the liver. Voxel shimming was executed to optimise the homogeneity of the magnetic field within the specific volume of interest. Two ${ }^{1} \mathrm{H}$ spectra were collected from the hepatic parenchyma in the same prescanning conditions using a point-resolved spectroscopy pulse sequence (interpulse delay repetition time $3000 \mathrm{~ms}$, spin echo time $25 \mathrm{~ms}, 1024$ data points over a 1000-Hz spectral width, 64 acquisitions) with and without suppression of the water signal respectively. Areas of resonances from protons of water (4.8 parts per million [ppm]) and methylene groups in fatty acids chains of the hepatic triglycerides $(1.4 \mathrm{ppm})$ were obtained with a time-domain non-linear fitting routine using commercial software (VARPRO-MRUI; available from www.mrui.uab.es; accessed 15 July 2005). The IHF percentage was calculated by dividing the integral of the methylene groups in fatty acid chains of the hepatic triglycerides by the sum of methylene groups and water and multiplying by 100 . This procedure was validated against the assessment of lipid content obtained by means of liver biopsies in humans [12].

\section{Analytical procedures}

Plasma glucose was measured with a Beckman glucose analyser [4]. Plasma NEFA and plasma total cholesterol, HDL cholesterol and triglycerides were measured as described previously [4]. LDL cholesterol was calculated using the Friedwald formula. Plasma free insulin was measured with a commercially available RIA kit (Linco Research, St Charles, MO, USA). Plasma leptin concentrations were determined as described previously [4] by RIA with a human kit (Linco Research). Plasma adiponectin was measured using a commercially available RIA kit (Linco Research) as described previously [4]. Plasma resistin was measured using a commercially available ELISA kit (BioVendor Laboratory Medicine, Brno, Czech Republic). $d_{2}$-Glucose enrichment was measured by GCMS as described previously [4].

\section{Calculations}

Glucose kinetics were calculated using Steele's equations for the non-steady state [13]. Steady state of plasma di- 
deuterated glucose enrichments was reached in the study groups during both the end of the equilibration period and the last $30 \mathrm{~min}$ of the insulin clamp. Endogenous glucose production was calculated by subtracting the glucose infusion rate from the rate of glucose appearance measured with the isotope tracer technique. Total body glucose uptake was determined during the clamp by adding the rate of residual endogenous glucose production to the rate of glucose disappearance. The metabolic clearance rate was calculated to normalise the glucose turnover rate to the plasma glucose concentration. Glucose, lipid and protein oxidations were estimated as described previously [14] in the postabsorptive state. During the insulin clamp, protein oxidation was corrected for changes in pool size [15]. Estimated portal venous insulin concentration was calculated assuming a constant portal-to-peripheral insulin gradient of 2.4:1 [16]. Estimated hepatic sinusoidal insulin concentration was calculated by assuming that hepatic sinusoid receives $80 \%$ of its blood supply from the portal vein and $20 \%$ from the hepatic artery [17].

\section{Statistical analysis}

Data are means \pm SD. Analyses were performed using SSPS (version 10.0; SPSS, Chicago, IL, USA). The steady state for plasma glucose $6,6-{ }^{2} \mathrm{H}_{2}$ enrichment was defined as a non-significant correlation with time $(p>0.05)$ using linear regression. Independent $t$-tests (two-tailed) were used to compare basal values between groups and a $p$ value less than 0.05 was considered statistically significant. Twofactor repeated measures ANOVA was used to assess group and insulin effects and group $\times$ insulin interactions with respect to the parameters of energy homeostasis, glucose and lipid metabolism during the clamp when appropriate. Two-tailed Pearson correlation analysis was performed to assess associations between variables.

\section{Results}

Anthropometric and laboratory characteristics

of study groups

Anthropometric parameters of study subjects are summarised in Table 1. Patients with diabetes and normal subjects were matched for age, sex, BMI and physical activity index. Postabsorptive plasma glucose was higher in patients with diabetes in comparison with normal subjects (Table 1). During the clamp, plasma glucose concentration was maintained at the fasting level in normal subjects, while in patients with diabetes it was clamped at a higher level ( $6.5 \pm 1.0$ vs $4.9 \pm 0.2 \mathrm{mmol} / \mathrm{l} ; p=0.046$; Fig. 1$)$. In patients with diabetes the therapeutic control of glucose homeostasis was poor, as reflected by the $\mathrm{HbA}_{1} \mathrm{c}$ level (Table 1). Plasma NEFA concentration was also higher in diabetic patients in comparison with normal subjects (Table 1) and during the clamp it dropped similarly in the two study groups but remained at a slightly higher level in diabetic patients in comparison with normal subjects $(0.12 \pm 0.03$ vs $0.08 \pm 0.02 \mathrm{mmol} / 1 ; p=0.051)$. Markers of kidney function and the lipid profile were not different between groups. Transaminases and $\gamma$-glutamyltransferase were within the normal range in all study subjects.

Glucose metabolism in the postabsorptive state and during the insulin clamp

The endogenous glucose production rate was increased in patients with type 1 diabetes with respect to normal subjects in the basal state $\left(4.50 \pm 1.28\right.$ vs $2.29 \pm 0.31 \mathrm{mg} \mathrm{kg}^{-1} \mathrm{~min}^{-1}$; $p<0.001)$ and during the insulin clamp $(1.38 \pm 1.13$ vs $0.20 \pm$ $\left.0.21 \mathrm{mg} \mathrm{kg}^{-1} \mathrm{~min}^{-1} ; p<0.01\right)$. During the clamp the diabetic patients showed a certain degree of insulin resistance with respect to the suppression of the endogenous glucose production (66 \pm 30 and $92 \pm 8 \%$ in diabetic and normal subjects respectively; $p=0.047$; Fig. $2 \mathrm{a}$ ). The metabolic clearance rate of glucose was also reduced in diabetic patients with respect to normal subjects $\left(4.3 \pm 1.3 \mathrm{vs} 6.0 \pm 1.6 \mathrm{mg} \mathrm{kg}^{-1}\right.$ $\min ^{-1} ; p<0.001$; Fig. 2b).

\section{IHF content}

${ }^{1}$ HMRS of the liver showed that the IHF content was reduced in patients with type 1 diabetes $(1.5 \pm 0.7 \%)$ with respect to normal subjects $(2.2 \pm 1.0 \% ; p<0.03)$ (Fig. 2c). Pearson's correlation analysis showed that the IHF content a

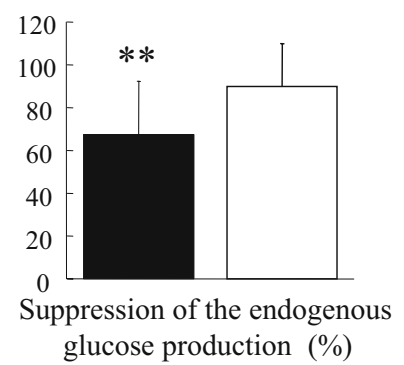

C

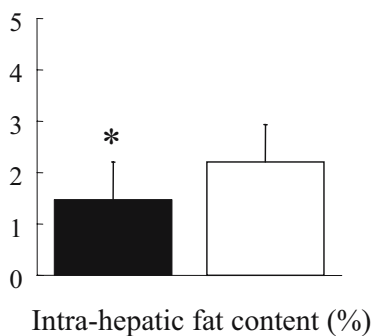

b

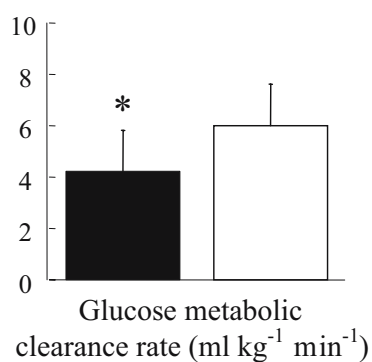

d

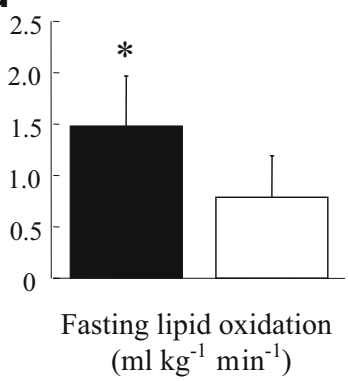

Fig. 2 Insulin-induced suppression of endogenous glucose production (a), glucose metabolic clearance rate (b), IHF content (c) and fasting lipid oxidation (d) in patients with type 1 diabetes (filled bars) and normal subjects (open bars). Means \pm SD. $* p<0.01$, ${ }^{* *} p<0.05$ vs normal subjects (independent two-tailed t-test) 
was not associated with parameters of peripheral and hepatic glucose disposal.

\section{Energy metabolism}

The resting energy expenditure was not different between diabetic patients and normal subjects $(1690 \pm 234$ vs $1707 \pm 308 \mathrm{kcal} /$ day; $p=0.86$ ) and no difference was found during the clamp $(1663 \pm 226$ vs $1650 \pm 445 \mathrm{kcal} /$ day in diabetic patients and normal subjects; $p=0.91$ ). The respiratory quotient was lower in patients with type 1 diabetes in comparison with normal subjects $(0.74 \pm 0.05$ vs $0.84 \pm 0.06$; $p<0.001$ ), reflecting greater fasting lipid oxidation in patients with diabetes in comparison with normal subjects (1.48 \pm 0.49 vs $0.79 \pm 0.40 \mathrm{mg} \mathrm{kg}^{-1} \mathrm{~min}^{-1} ; p<0.001$; Fig. $2 \mathrm{~d}$ ). During the clamp the respiratory quotient increased in both groups $(0.83 \pm 0.06$ vs $0.90 \pm 0.05$ in diabetic patients and normal subjects respectively) but remained higher in normal subjects $(p=0.045)$. In association with this, similar suppression of lipid oxidation was observed in study groups $(44 \pm 20$ and $58 \pm 21 \%$ in diabetic patients and normal subjects respectively); despite this, lipid oxidation remained higher in diabetic patients than in normal subjects $\left(0.91 \pm 0.51\right.$ vs $\left.0.35 \pm 0.19 \mathrm{mg} \mathrm{kg}^{-1} \min ^{-1} ; p<0.001\right)$.

\section{Endocrine profile}

Fasting plasma insulin was not different between study groups (Table 2). During the clamp, plasma insulin concentration was also not different between groups $(385 \pm 83$ and $366 \pm 21 \mathrm{pmol} / 1$ in diabetic patients and normal subjects respectively; $p=0.238$ ). The estimated hepatic insulin concentration in the fasting state was lower in diabetic patients than in normal subjects (Table 2). Fasting glucagon concentration was not different between study groups (Table 2). On the basis of the concentrations given above, the glucagon/estimated hepatic insulin ratio was increased in type 1 diabetic patients when compared with normal subjects ( $p=0.047$; Table 2). Concentrations of the plasma adipocyte-derived peptides leptin and resistin were not

Table 2 Endocrine profile of study groups

\begin{tabular}{llc}
\hline & $\begin{array}{l}\text { Type 1 diabetes } \\
\text { mellitus }\end{array}$ & $\begin{array}{c}\text { Normal } \\
\text { subjects }\end{array}$ \\
\hline $\begin{array}{l}\text { Insulin (pmol/l) } \\
\text { Estimated hepatic insulin }\end{array}$ & $62 \pm 46$ & $44 \pm 13$ \\
$\quad 60 \pm 30^{\mathrm{a}}$ & $94 \pm 27$ \\
(pmol/1) & $81 \pm 52$ & \\
Glucagon (pg/ml) & $1.7 \pm 1.2^{\mathrm{a}}$ & $04 \pm 21$ \\
hepatic insulin ratio & & $0.9 \pm 0.4$ \\
Adiponectin $(\mu \mathrm{g} / \mathrm{ml})$ & $15 \pm 6$ & $7 \pm 4$ \\
Leptin $(\mathrm{ng} / \mathrm{ml})$ & $3.2 \pm 2.2$ & $5.2 \pm 3.3$ \\
Resistin $(\mathrm{ng} / \mathrm{ml})$ & $3.3 \pm 0.8$ & $3.2 \pm 0.8$ \\
\hline
\end{tabular}

${ }^{\mathrm{a}} p<0.05$ vs normal subjects (independent two-tailed $t$-test) different between study groups; in contrast, plasma adiponectin concentration was higher in diabetic patients than in normal subjects (Table 2).

\section{Discussion}

The present study demonstrates that in moderately insulinresistant patients with type 1 diabetes the IHF content is reduced and is not associated with insulin resistance. In these patients the reduced IHF content is associated with markers of enhanced whole-body fat oxidation, possibly due to the local effects of the higher glucagon/hepatic insulin ratio on liver substrate disposal.

Insulin resistance characterises type 2 diabetes but several studies have shown that a certain degree of insulin resistance is also present in patients with type 1 diabetes [18], especially when metabolic control is poor [19]. In agreement with the hypothesis that ectopic fat accumulation may be related to impaired insulin action, an increased IMCL content in association with peripheral insulin resistance was reported in patients with type 1 diabetes as well as in patients with type 2 diabetes and their relatives [4]. More recently it was suggested that ectopic fat accumulation within the liver may be also associated with peripheral and hepatic insulin resistance in nondiabetic individuals $[5,6]$ and is common in obese patients with type 2 diabetes [20]. Some authors suggest that fatty liver represents a body composition manifestation of obesity and visceral adiposity in insulin-resistant subjects [21], but at the same time they suggest that the impact on insulin sensitivity is determined regardless of intra-abdominal and overall adiposity [6]. Moreover, fatty liver accumulation appears to be peculiarly associated with hepatic insulin resistance; in fact moderate weight reduction in obese patients with type 2 diabetes was associated with reduced IHF content and improvement of hepatic insulin sensitivity but no change in insulin-stimulated peripheral glucose metabolism [22]. In the present study we report that in patients with type 1 diabetes and poor metabolic control in whom mild hepatic (Fig. 2a) and moderate peripheral insulin resistance (Fig. 2b) was detected, the IHF content (Fig. 2c) was reduced rather than increased in comparison with normal subjects. In patients with type 1 diabetes, variables other than anthropometric features and insulin action may affect the IHF content. On the basis of the increased mobilisation of NEFA from the adipose tissue (Table 1) and the increased glucagon/estimated hepatic insulin ratio (Table 2) characterising our patients with type 1 diabetes, we hypothesised that the capacity of their livers to oxidise fatty acids might be higher, with a possible consequent impact on the IHF content. Confirming this hypothesis, we found that the resting energy expenditure was not different between groups during both the fasting and the insulin-stimulated states, but the contribution of lipid oxidation was significantly increased in patients with type 1 diabetes, as reflected by the respiratory quotient and the lipid oxidation rates (Fig. 2d). It is likely that this pattern of resting energy 
substrate oxidation, also reported by other authors [7-9], may be determined by the unbalanced relative concentrations of hepatic glucagon and insulin. It is well known that elevation of the hepatic glucagon/insulin ratio activates the carnitine acyltransferase reaction, the first step in the oxidation of fatty acids, allowing the entrance of long-chain fatty acyl-CoA into the mitochondrion [23]. We suggest that in the everyday life of patients with type 1 diabetes with poor metabolic control the increased plasma concentration of NEFA, along with the increased plasma glucose and amino acid [24] concentrations, induces a nutritionally plentiful condition. At the level of the peripheral tissues, i.e. skeletal muscle, this increased flux of nutrients through the tricarboxylic acid cycle induces increments in the cytoplasmic level of malonyl-CoA, which is known to be a potent allosteric inhibitor of carnitine-palmitoyl-transferase-1 (CPT-1), and hence impairs the intramitochondrial transport of long-chain fatty acyl-CoA and limits their oxidative disposal. Intramyocellular accumulation of these triglyceride precursors would eventually push IMCL accumulation, as we have shown previously [4], with a mechanism similar to what may be hypothesised for the skeletal muscle of insulin-resistant individuals with type 2 diabetes [25]. The increased IMCL content or the increased intramyocellular content of a specific fatty acid metabolite would mediate the development of muscular insulin resistance as a mechanistic defence against the increased nutritional flux. In contrast, at the level of the liver the flux of long-chain fatty acyl-CoA through the tricarboxylic acid cycle would be sustained despite nutrient excess, because of the permissive effect of the high hepatic glucagon/ insulin ratio in activating the hepatocytes' CPT-1 reaction in patients with type 1 diabetes, but not in those with type 2 diabetes. This view would explain why type 1 diabetes is a condition in which ectopic fat accumulation is limited to the skeletal muscle, as we have shown previously [4], sparing the liver site; this ectopic fat accumulation in muscle may be sufficient to trigger peripheral insulin resistance, which may be present despite the lack of ectopic fat accumulation within the liver. Type 1 diabetes would therefore represent a peculiar condition in which the impact of oxidative disposal of substrates on the IHF content may be more profound than in type 2 diabetes, probably because of the unbalanced glucagon and insulin concentrations at the hepatic site. Supporting this hypothesis, Bugianesi et al. [26] have recently shown that increased NEFA flux may induce fatty liver infiltration despite greater whole-body lipid oxidation in insulin-resistant but non-diabetic individuals, in whom the portoperipheral insulin gradient and relative glucagon concentrations are not affected as they are in type 1 diabetic patients. This hypothesis clearly implies that the increased whole-body lipid oxidation reported in the present work in patients with type 1 diabetes is largely attributable to a feature of the liver and not to a skeletal muscle feature. It should be kept in mind that in this study lipid oxidation was assessed using indirect calorimetry, reflecting whole-body substrate oxidation. We cannot exclude the possibility that the association between lipid oxidation and IHF content would have been stronger if more specific measures had been employed.

The finding of depleted liver fat content in patients with type 1 diabetes is remarkably similar to the finding of depleted liver glycogen stores in patients with similar features $[27,28]$. In this regard, we need to emphasise that in the present study the patients were studied as they were in their everyday life (poor metabolic control), and therefore it is likely that these findings do not represent an intrinsic alteration but rather that they may be due to the poor therapeutic control. This view is supported by the fact that in type 1 diabetic patients with long-term near-normoglycaemia $\left(\mathrm{HbA}_{1} \mathrm{c}<7 \%\right.$ for 1 year), hepatic glycogen synthesis was normalised after ingestion of a mixed meal in association with restoration of the glucagon/estimated hepatic insulin ratio [29]. Insulin and glucagon are considered to be of pivotal importance in the regulation of hepatic glycogen synthesis and turnover in humans [30]. We would expect a similar behaviour for the IHF content, and we are in the process of testing this hypothesis by measuring the long-term effects of intrahepatic pancreatic islet transplantation on the IHF content in individuals with type 1 diabetes.

Since studies have also implicated adipocyte-derived hormones in causing insulin resistance, modulating ectopic fat accumulation and possibly fat oxidation, we measured plasma concentrations of leptin, adiponectin and resistin in the fasting condition (Table 2). Only the adiponectin concentration was different from that in normal subjects, confirming our previous report [4], although we failed to find a significant association with the IHF content.

In conclusion, the present study demonstrates that in moderately insulin-resistant patients with type 1 diabetes the IHF content is reduced. Therefore, it cannot be considered a mediator of peripheral and hepatic insulin resistance in this condition, as suggested, in contrast to patients with type 2 diabetes. We propose that the enhanced wholebody fat oxidation found in these patients, which is probably due to the unbalanced hepatic glucagon and insulin concentrations, may contribute to the lower IHF content. This supports the potential role of a defect of liver fat oxidation in the pathogenesis of non-alcoholic fatty liver disease and highlights the therapeutic potential of the modulation of hepatic fatty acid oxidation.

Acknowledgement This study was supported by grants from Telethon (JT-01) and the Juvenile Diabetes Research Foundation (JT01) within the Telethon-JDRF Center for Beta Cell Replacement, and by the Italian Minister of Health (RF98.49, RF99.55, RF01.1831).

\section{References}

1. McGarry JD (1992) What if Minkowski had been ageusic? An alternative angle on diabetes. Science 258:766-770

2. Krssak M, Falk Petersen K, Dresner A et al. (1999) Intramyocellular lipid concentrations are correlated with insulin sensitivity in humans: a ${ }^{1} \mathrm{H}$ NMR spectroscopy study. Diabetologia 42:113-116 
3. Perseghin G, Scifo P, De Cobelli F et al. (1999) Intramyocellular triglyceride content is a determinant of in vivo insulin resistance in humans: ${ }^{1} \mathrm{H}^{-13} \mathrm{C}$ NMR spectroscopy assessment in offspring of type 2 diabetic parents. Diabetes 48:1600-1606

4. Perseghin G, Lattuada G, Danna M et al. (2003) Insulin resistance, intramyocellular lipid content and plasma adiponectin concentrations in patients with type 1 diabetes. Am J Physiol Endocrinol Metab 285:E1174-E1181

5. Marchesini G, Brizi M, Bianchi G et al. (2001) Nonalcoholic fatty liver disease: a feature of the metabolic syndrome. Diabetes 50:1844-1850

6. Seppala-Lindroos A, Vehkavaara S, Hakkinen A-M et al. (2002) Fat accumulation in the liver is associated with defects in insulin suppression of glucose production and serum free fatty acids independent of obesity an normal men. J Clin Endocrinol Metab 87:3023-3028

7. Wohl P, Wohl P, Girman P, Pelikanova T (2004) Inflexibility of energy substrate oxidation in type 1 diabetic patients. Metabolism 53:655-659

8. Raguso CA, Coggan AR, Gastaldelli A, Sidossis LS, Bastyr EJ III, Wolfe RR (1995) Lipid and carbohydrate metabolism in IDDM during moderate and intense exercise. Diabetes 44: 1066-1074

9. Tuominen JA, Ebeling P, Vuorinen-Markkola H, Koivisto VA (1997) Post-marathon paradox in IDDM: unchanged insulin sensitivity in spite of glycogen depletion. Diabet Med 14:301-308

10. Baecke JAH, Burema J, Frijters JER (1982) A short questionnaire for the measurement of habitual physical activity in epidemiological studies. Am J Clin Nutr 36:936-942

11. Hother-Nielsen O, Beck-Nielsen H (1990) On the determination of basal glucose production rate in patients with type 2 (noninsulin-dependent) diabetes mellitus using primed-continuous 3- ${ }^{3} \mathrm{H}$-glucose infusion. Diabetologia 33:603-610

12. Thomsen $\mathrm{C}$, Becker U, Winkler K, Christoffersen P, Jensen M, Henriksen O (1994) Quantification of liver fat by using magnetic resonance spectroscopy. Magn Reson Imaging 12:487-495

13. Steele R (1959) Influence of glucose loading and of injected insulin on hepatic glucose output. Ann NY Acad Sci 82:420-431

14. Perseghin G, Scifo P, Danna M et al. (2002) Normal insulin sensitivity and IMCL content in overweight humans are associated with higher fasting lipid oxidation. Am J Physiol Endocrinol Metab 283:E556-E564

15. Tappy L, Owen OE, Boden G (1988) Effect of hyperinsulinemia on urea pool size and substrate oxidation rates. Diabetes $37: 1212-1216$

16. Hother-Nielsen O, Schmitz O, Bak J, Beck-Nielsen H (1987) Enhanced hepatic insulin sensitivity, but peripheral insulin resistance in patients with type 1 (insulin-dependent) diabetes. Diabetologia 30:834-840
17. Adkins-Marshall B, Pagliassotti MJ, Asher JR et al. (1992) Role of hepatic nerves in response of liver to intraportal glucose delivery in dogs. Am J Physiol Endocrinol Metab 262:E679E686

18. De Fronzo RA, Simonson D, Ferrannini E (1982) Hepatic and peripheral insulin resistance: a common feature of type 2 (noninsulin dependent) and type 1 (insulin-dependent) diabetes mellitus. Diabetologia 23:313-319

19. Lager I, Lönnroth P, Von Schenck H, Smith U (1983) Reversal of insulin resistance in type 1 diabetes after treatment with continuous subcutaneous insulin infusion. Br Med J 287:1661-1664

20. Kelley DE, McKolanis TM, Hegazi RA, Kuller LH, Kalhan SC (2003) Fatty liver in type 2 diabetes mellitus: relation to regional adiposity, fatty acids, and insulin resistance. Am J Physiol Endocrinol Metab 285:E906-E916

21. Pietilainen KH, Rissanen A, Kaprio J et al. (2005) Acquired obesity is associated with increased liver fat, intra-abdominal fat, and insulin resistance in young adult monozygotic twins. Am J Physiol Endocrinol Metab 288:E768-E774

22. Petersen KF, Dufour S, Befroy D, Lehrke M, Hendler RE, Shulman GI (2005) Reversal of nonalcoholic hepatic steatosis, hepatic insulin resistance, and hyperglycaemia by moderate weight reduction in patients with type 2 diabetes. Diabetes 54:603-608

23. McGarry JD, Foster DW (1977) Hormonal control of ketogenesis. Biochemical considerations. Arch Intern Med 137:495-501

24. Luzi L, Castellino P, Simonson DC, Petrides AS, DeFronzo RA (1990) Leucine metabolism in IDDM. Role of insulin and substrate availability. Diabetes 39:38-48

25. Ruderman N, Prentki M (2004) AMP kinase and malonyl-CoA: targets for therapy of the metabolic syndrome. Nat Rev Drug Discov 3:340-351

26. Bugianesi E, Gastaldelli A, Vanni E et al. (2005) Insulin resistance in non-diabetic patients with non-alcoholic fatty liver disease: sites and mechanisms. Diabetologia 48:634-642

27. Hwang JH, Perseghin G, Rothman DL et al. (1995) Impaired net hepatic glycogen synthesis in insulin-dependent diabetic subjects during mixed meal ingestion. ${ }^{13} \mathrm{C}$ nuclear magnetic resonance spectroscopy study. J Clin Invest 95:783-787

28. Bischof MG, Krssak M, Krebs M et al. (2001) Effects of shortterm improvement of insulin treatment and glycaemia on hepatic glycogen metabolism in type 1 diabetes. Diabetes 50:392-398

29. Bischof MG, Bernroider E, Krssak M et al. (2002) Hepatic glycogen metabolism in type 1 diabetes after long-term near normoglycaemia. Diabetes 51:49-54

30. Roden M, Perseghin G, Petersen KF et al. (1996) The roles of insulin and glucagon in the regulation of hepatic glycogen synthesis and turnover in humans. J Clin Invest 97:642-648 